\title{
A broken heart in a broken car
}

\author{
Susanne Anna Schlossbauer, Jelena-Rima Ghadri, Victoria Lucia Cammann, \\ Willibald Maier, Thomas F. Lüscher, Christian Templin
}

Department of Cardiology, University Heart Center, University Hospital Zurich, Zurich, Switzerland

\begin{abstract}
Takotsubo syndrome (TTS) is still a relatively understudied and often undetected disease. It is usually preceded by emotional or physical triggers. We here report a case of TTS following a car accident. Typical apical ballooning with moderate reduction of left ventricular ejection fraction (LVEF) and increased level of pro-B-type natriuretic peptide (BNP) as well as slightly increased creatine kinase and troponin T values were found in this 76-year-old female patient, $6 \mathrm{~h}$ after a car accident. At 10 weeks follow-up, we observed a normalization of regional wall motion, LVEF, electrocardiogram and pro-BNP. TTS is an acute heart failure syndrome and an important differential diagnosis of acute coronary syndrome. (Cardiol J 2016; 23, 3: 352-354)
\end{abstract}

Key words: takotsubo syndrome, broken heart syndrome, apical ballooning

Takotsubo syndrome (TTS) is characterized by distinct wall motion abnormalities and symptoms of heart failure [1,2]. Elderly, postmenopausal women are mainly predisposed to TTS and they typically present with symptoms of acute chest pain, dyspnea or even cardiogenic shock. TTS is preceded in most cases by emotional or physical triggers and sometimes a combination of both. However, TTS can also occur in the absence of any trigger [3].

A 76-year-old lady presented to the Emergency Department $6 \mathrm{~h}$ after a car accident. A truck crashed laterally into her car with a velocity of approximately $30 \mathrm{mph}$, causing a rear-end collision. She was wearing a seat belt and reported to have not bounded against the steering wheel with her chest. The airbags opened and hit her from laterally dorsal. Immediately after the crash, the patient felt a heavy central chest pressure irradiating to the left. Then, she drove back home on her own and took ibuprofen for her chest pain.

Due to persisting symptoms she decided to go to the Emergency Department. Clinical examination showed a beginning superficial hematoma on the left lateral chest without instability. Her chest X-ray was normal and admission 12-lead electrocardiogram (ECG) demonstrated no signs of acute ischemia. Laboratory findings, however revealed elevated high-sensitive troponin $\mathrm{T}(431 \mathrm{ng} / \mathrm{L}$, normal < $14 \mathrm{ng} / \mathrm{L})$, creatine kinase $(\mathrm{CK}, 239 \mathrm{U} / \mathrm{L}$, normal $<170 \mathrm{U} / \mathrm{L})$ and pro-B-type natriuretic peptide (BNP, $1965 \mathrm{ng} / \mathrm{L}$, normal $<738 \mathrm{ng} / \mathrm{L}$ ). Given the elevated cardiac enzymes and chest discomfort the patient had the presumptive diagnosis of acute coronary syndrome (ACS).

In order to exclude the presence of pericardial effusion after chest trauma, emergency echocardiography was performed, demonstrating a moderately reduced left ventricular ejection fraction (LVEF) of 39\% with an apical ballooning pattern; a moderate mitral valve insufficiency, elevated systolic pulmonary artery pressure (PASP) of $52 \mathrm{~mm} \mathrm{Hg}$, and minimal pericardial effusion without hemodynamic consequence.

Interestingly coronary angiography showed normal coronary arteries, but left ventricular angiography confirmed apical ballooning with a decreased LVEF as seen by echocardiography (Fig. 1, supplementary video - see journal website: www.cardiologyjournal.org). The patient was

Address for correspondence: Christian Templin, MD, PhD, University Hospital Zurich, University Heart Center, Department of Cardiology, Rämistr. 100, 8091 Zurich, Switzerland, e-mail: Christian.Templin@usz.ch 


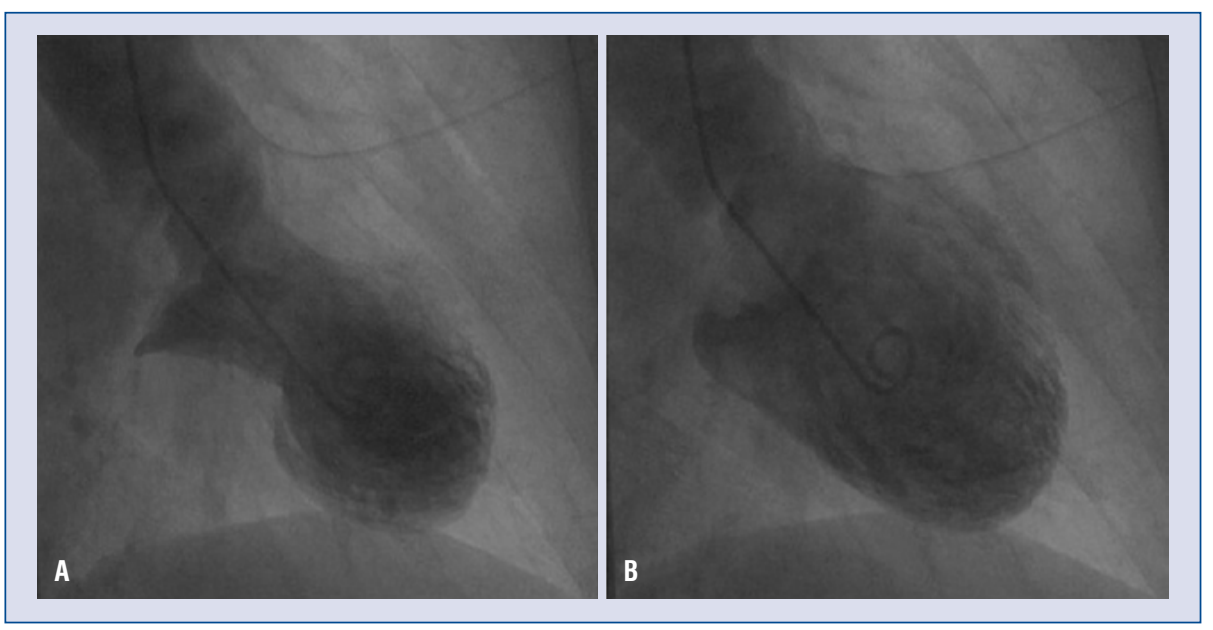

Figure 1. Left ventricular angiograms during systole (A) and diastole (B) showing apical ballooning pattern.

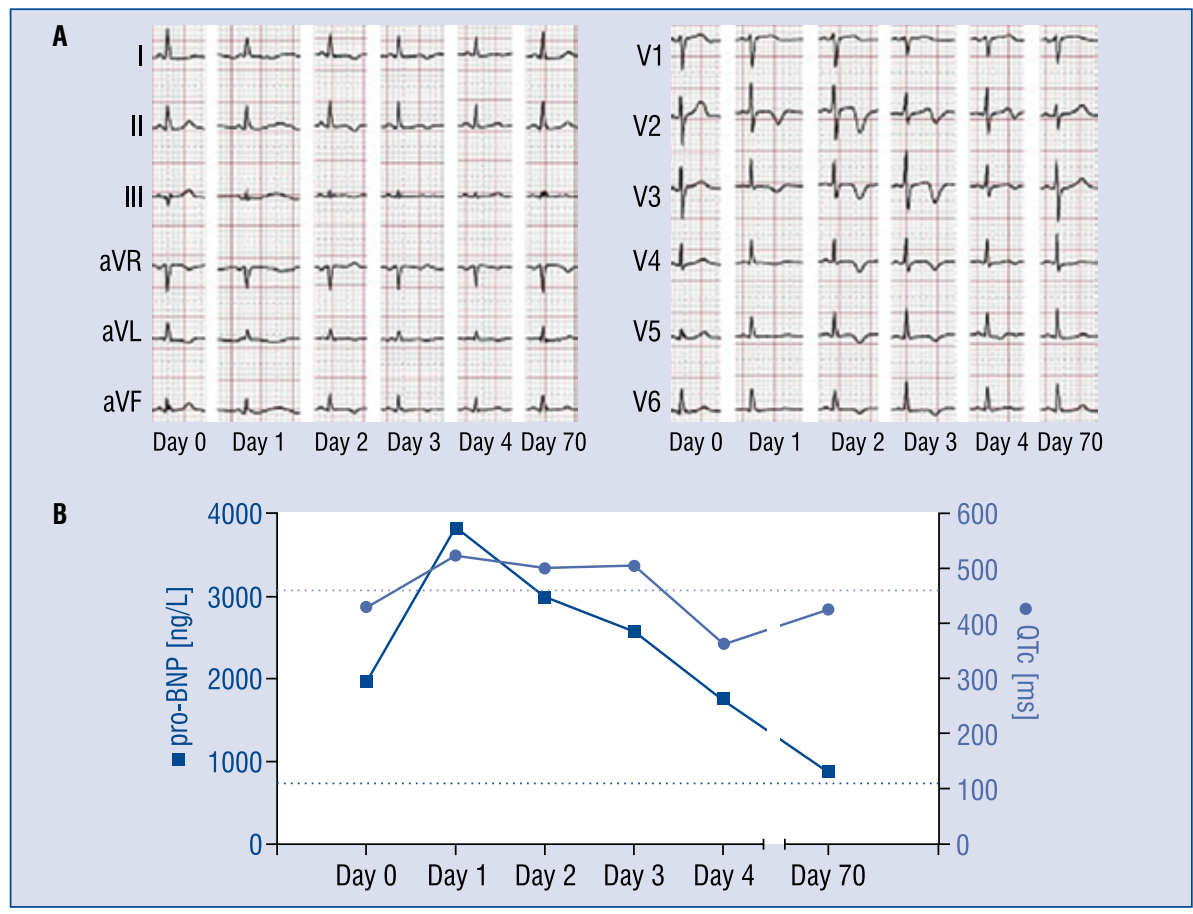

Figure 2. Pro-B-type natriuretic peptide (BNP), QT-time corrected for heart rate (QTc) and electrocardiogram (ECG) changes overtime from admission to follow-up visit (day 70 after index event). At day 70, QTc, pro-BNP, ECG and left ventricular ejection fraction had returned to normal; $A$. ECG changes: on the right limb leads, on the left chest leads. T-inversion is most pronounced at day 2; B. QTc and pro-BNP values reached their maximum at day 1.

then transferred to the coronary care unit for monitoring and was put on an angiotensin converting enzyme (ACE) inhibitor and beta-blocker.

The next day, ECG showed new deep T-inversions in leads V2 and V3. On the second day T-inversions extended to leads V4-V6, inferior and lateral leads. From day 3, T inversions regressed and returned normal by day 4 (Fig. 2A). In addition a prolonged QTc with a maximum of $524 \mathrm{~ms}$ on day 1 after the index event was noted, and returned normal at day $6(397 \mathrm{~ms})$. Similar to QTc, pro-BNP reached its maximum value at day 1 , and decreased 
then gradually (Fig. 2B). High sensitive troponin T and CK continuously declined over days.

Follow-up echocardiography at day 6 after the index event showed a slight improvement of LVEF (49\%), but remaining hypokinesia of the midventricular segments and apical akinesia; PASP returned normal $(32 \mathrm{~mm} \mathrm{Hg}$ ) and mitral valve regurgitation showed a mild improvement. The patient was discharged with ACE inhibitor, betablocker and a diuretic.

Ten weeks follow up visit revealed normal LVEF (61\%) without regional wall motion abnormalities and a significant decrease of pro-BNP to almost normal levels (873 ng/L) (Fig. 2B).

Although TTS has been described a quarter of a century ago, its pathophysiology still remains elusive. It is clear though, that based on symptoms [4], laboratory and ECG findings [5], TTS can be considered an ACS [6] often associated with signs and symptoms of acute heart failure. Of note, TTS is often preceded by an emotional or physical trigger prior to the acute event.

So far different ECG patterns have been described in TTS and Mitsuma et al. [7] have elaborated four phases of ECG changes over time, including ST-segment elevation as phase I immediately after symptom onset, negative T-waves in phase II (day 1-3), reversion of negative $T$ waves as phase III (day 2-6) and evolution of giant negative $T$ waves seen in phase IV, up to 60 days after symptom onset. Late presentation after symptom onset was considered as a possible reason for detection of ST-segment elevation in about $30-60 \%$ of patients with TTS $[3,7]$.

The case outlined here demonstrated an ECG feature upon admission that did neither show a J-wave in the early phase, as described by Zorzi et al. [8], nor ST-segment elevation. The relatively late presentation of $6 \mathrm{~h}$ after symptom onset might have been the reason for the missing documentation of these transient ECG changes.

We noted a typical T-wave inversion on day 1 , reaching its maximum with an amplitude of $\geq 3 \mathrm{~mm}$ and including $\geq 6$ leads at day 2 , later followed by a reversion of negative $T$ wave starting on day 3 , consistent with reports from Mitsuma et al. [7] and Looi et al. [9]. No giant negative T-wave was observed subsequently. Consistently to what was reported by Bennett et al. [10], a maximum QTc prolongation within $24 \mathrm{~h}$ after symptom onset was documented in this female patient as well.
Furthermore we observed a typical increase of pro-BNP up to a maximum $24 \mathrm{~h}$ after clinical symptom onset, with a subsequent decrease in LVEF that returned back to normal later on.

Takotsubo syndrome is still a relatively understudied and often undetected disease. Clinicians have to keep in mind that it is also an important differential diagnosis of ACS and can mimic features of a non-ST elevation myocardial infarction. Our case is a classic example of a typical TTS patient presenting to the Emergency Department, i.e. of female gender and of postmenopausal age with a preceding stressful event. ECG and laboratory values may manifest differently within their first clinical presentation, however laboratory markers and ECG show a classical course.

\section{Conflict of interest: None declared}

\section{References}

1. Hurst RT, Prasad A, Askew JW, Sengupta PP, Tajik AJ. Takotsubo cardiomyopathy: A unique cardiomyopathy with variable ventricular morphology. J Am Coll Cardiol Cardiovasc Imag, 2010; 3: 641-964.

2. Ghadri JR, Ruschitzka F, Luscher TF, Templin C. Takotsubo cardiomyopathy: Still much more to learn. Heart, 2014; 100: 1804-1812.

3. Templin C, Ghadri JR, Diekmann J et al. Clinical features and outcomes of takotsubo (stress) cardiomyopathy. N Engl J Med, 2015; 373: 929-938.

4. Prasad A, Lerman A, Rihal CS. Apical ballooning syndrome (Tako-Tsubo or stress cardiomyopathy): A mimic of acute myocardial infarction. Am Heart J, 2008;155:408-417.

5. Desmet WJ, Adriaenssens BF, Dens JA. Apical ballooning of the left ventricle: First series in white patients. Heart, 2003; 89: 1027-1031.

6. Lüscher TF, Templin C. Is takotsubo syndrome a microvascular acute coronary heart syndrome? Towards of a new definition. Eur Heart J, 2016; in press.

7. Mitsuma W, Kodama M, Ito M et al. Serial electrocardiographic findings in women with Takotsubo cardiomyopathy. Am J Cardiol, 2007; 100: 106-109.

8. Zorzi A, Migliore F, Perazzolo Marra M, Tarantini G, Iliceto S, Corrado D. Electrocardiographic J waves as a hyperacute sign of Takotsubo syndrome. J Electrocardiol, 2012; 45: 353-356.

9. Looi JL, Wong CW, Lee M, Khan A, Webster M, Kerr AJ. Usefulness of ECG to differentiate Takotsubo cardiomyopathy from acute coronary syndrome. Int J Cardiol, 2015; 199: 132-140.

10. Bennett J, Ferdinande B, Kayaert P et al. Time course of electrocardiographic changes in transient left ventricular ballooning syndrome. Int J Cardiol, 2013; 169: 276-280. 